\title{
C-PMSE - Improved Spectrum Utilization and Coexistence by Cognitive PMSE Systems
}

\author{
A Strategic Application-oriented Research \& Development Project in Germany
}

\author{
Uwe Beutnagel-Buchner \\ Frequency Affairs \\ Robert Bosch GmbH \\ Gerlingen, Germany \\ uwe.beutnagel-buchner@de.bosch.com
}

\author{
Norbert Hilbich \\ Application Engineering \\ Sennheiser Electronic GmbH \& Co. KG \\ Wennebostel, Germany \\ norbert.hilbich@sennheiser.com
}

\author{
Andreas Wilzeck \\ Institute of Communications Technology \\ Leibniz Universität Hannover \\ Hannover, Germany \\ andreas.wilzeck@ikt.uni-hannover.de
}

\begin{abstract}
This contribution provides an overview of the German R\&D project C-PMSE, which aims to develop a research platform for the evaluation and validation of Cognitive Radio (CR) procedures facilitating an improved coexistence of Program Making and Special Event (PMSE) systems in heterogeneous wireless scenarios. The platform will enable realistic coexistence studies with current and upcoming wireless standards and services in the television (TV) bands. Further, objectives of C-PMSE are to develop adequate channel and interference models, to study and verify multi-sensor, multilocation concepts in signal detection, signal classification, reactive and proactive radio resource management, and a local spectrum portfolio management. The fundamental concept is based on the ETSI STF 386: Methods, parameters and test procedures for cognitive interference mitigation techniques for use by PMSE devices [1]. The research is conducted in strong interaction with standardization and regulation. Hence, this contribution briefly discusses the PMSE related regulation activities, trends, and strategies in Europe.
\end{abstract}

Keywords - PMSE; coexistence; cognitive radio; wireless microphones; field trial

\section{INTRODUCTION}

All wireless systems share a common resource, the frequency spectrum; therefore, the access to the frequency spectrum needs to be organized so conflicts among different parties (users, services, technologies) are avoided. The ruling to access the frequency spectrum is a subject of national and international frequency regulation. Generally, one can notice dramatic changes in the frequency regulation in the recent years. The changes include changed frequency assignments due to the so-called digital dividend (switch from analogue television broadcasting to digital standards) and the discussion of approaches to enable a local and flexible frequency regulation, which is tailored to the particular needs of a service $[2],[3],[4],[5]$. One of the main reasons for the changes is the spectrum scarcity especially already encountered or foreseen for mobile and nomadic wireless communications caused by higher data-rate demands of new mobile services and applications (e.g. video telephony, mobile internet, mobile gaming, mobile television) [2],[3]. Relevant in this context is also the necessity to provide broadband Internet access to people living in rural areas [4],[6].

PMSE components and systems are used for professional event productions, e.g. at concerts, conferences, and sport events, but as well as audio and/or video conferencing systems. Typical application examples for PMSE are productions, recordings, live shows, reportages and electronic news gathering. The Professional Audio Manufacturers Alliance calculated in the year 2008 that approx. six million wireless PMSE routes are used in Europe. PMSE applications in the EU support directly or indirectly economic activities representing a turnover above 38 billion EUR [7]. Used devices include video cameras, microphones, in-ear monitors, intercom devices, and several data links, e.g. for remote control of light systems. Hence, many PMSE applications deal with the production of high quality source data of the (multimedia) content distributed later via media, broadcasting, and Internet.

Most of the audio links and more and more video and data links employed in PMSE applications are wireless, which of course requires a certain amount of frequency spectrum and a guaranteed high quality of services. A distortion-free transmission with very low latency is in case of many PMSE applications the challenge to solve - no matter if the transmission system is realized by digital or analogue technology [1].

The C-PMSE project will build a research platform: two lab and one field trial platform, which will be based on a fixed installation of multiple sensors distributed among different locations and buildings of the fair grounds of "Messe Berlin". The fundamental concept is based on the proposals developed within the ETSI STF 386 (see ETSI TS 102 800). Objectives are to evaluate and validate models, approaches and cognitive procedures in a heterogeneous coexistence scenario, which might be the result from a transition of the frequency regulation towards dynamic spectrum access schemes including hierarchical or priority-based spectrum assignments for kinds of services coexisting in the UHF band. Aim is to reach improved spectrum utilization and a coordinated coexistence of heterogeneous wireless services and systems in a given area (e.g. exhibition center, concert hall, fair grounds) allowing a 
distortion free operation of PMSE applications in the TV bands also in future.

The contribution is structured as follows: Section II outlines the current frequency regulation activities, strategies, and trends relevant to future PMSE systems and applications, based on cognitive radio procedures. Section III introduces the CPMSE project by providing an overview on its consortium, aims and objectives. Furthermore, the overall concept of the research platform, which will be developed and installed within the project, will be introduced. An outlook and a conclusion are given in Section IV.

\section{FREQUENCY REGULATION AND STANDARIZATION RELEVANT TO PMSE SYSTEMS \& APPLICATIONS}

In Europe, one important milestone for the European wide coordination of radio spectrum utilization was in 2002, when the European Union (EU) launched a regulatory framework for electronic communication, which was designed to promote European competitiveness and support world-class communications and broadcasting infrastructure in Europe. In the following, two complementary bodies were set up to facilitate consultation and to develop and support radio spectrum policy, the Radio Spectrum Policy Group (RSPG) with high-level representatives that advise on the broad policy in this area, and the Radio Spectrum Committee (RSC) which assists the European Commission (EC) in developing technical implementation measures. The EC, on the other hand, issues mandates to the European Conference of Postal and Telecommunications Administrations (CEPT) for the development of technical implementing measures.

Later on, a review of the regulatory framework led to a new instrument, which enables the European Commission to submit legislative proposals to the European Parliament and Council for establishing multi-annual Radio Spectrum Policy Programmes (RSPP). The RSPP intends to outline at a strategic level how the use of spectrum can contribute to the most important political objectives of the EU in a certain time frame. The goal is to set general regulatory principles and policy objectives to be applied for spectrum utilization in all sectors of the European market. Further on, it should define actions and common principles to enhance spectral efficiency and flexibility.

In 2010 the first RSPP has been setup and will determine step by step, until 2015, how spectrum use can contribute to EU objectives and optimize social, economic, and environmental benefits. The RSPP intends to follow a marketbased approach and is of special interest for PMSE industry and stakeholders, because one major focus will be on the spectrum of $300 \mathrm{MHz}-3 \mathrm{GHz}$, which addresses the currently primary spectrum for PMSE, $470-862 \mathrm{MHz}$. It covers the reallocation of the old analogue TV bands as well as the L band from $1452-1479.5 \mathrm{MHz}$, which could be an alternative spectrum to be used by PMSE European wide. One other important focus of RSPP is to collect and coordinate European inputs for agenda items for the next World Radio Conferences, particularly for the next one in 2012 (WRC-12).

The World Radio Conference WRC-07 decided to place two agenda items onto the WRC-12 agenda, which are of high interest for PMSE and cognitive systems generally. Agenda item 1.5 addresses "Harmonized Spectrum for Electronic news gathering (ENG)-RES COM 6/5“ and agenda item 1.19 should consider "regulatory measures and their relevance, to enable the introduction of software-defined radio and cognitive radio systems, based on the results of ITU-R studies, in accordance with Resolution 956 (WRC-07)".

In Europe, two Conference Preparatory Groups (CPG) are collecting and discussing inputs for WRC-12, CPG-A and CPG-B of the Electronic Communications Committee (ECC), which is part of the European Conference of Postal and Telecommunications Administrations (CEPT). They again, tasked working groups of ECC to support by technical inputs, for example, to investigate and define technical and operational requirements for the operation of cognitive radio systems.

Relevant working groups inside ECC that need to be addressed for regulation aspects on both, Cognitive Radio (CR) and PMSE, is the Frequency Management Working Groups 45 \& SRD_MG (WG FM 45/SRD_MG), the Spectrum 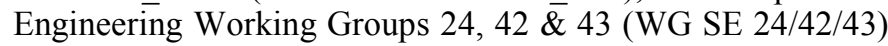 as well as the Regulatory Affairs Working Group WS_CR (WG RA WS_CR). In parallel to these activities, the Radio Spectrum Policy Group (RSPG) of the European Commission is working on opinions on CR.

Standardization in Europe for CR and PMSE is prepared in a first instance at the European Telecommunications Standards Institute (ETSI). Important existing standards describing technical characteristics and methods of measurement for PMSE are ETSI EN 300357 for "Cordless audio devices in the range $25 \mathrm{MHz}$ to $2.000 \mathrm{MHz}$ " and ETSI EN 300422 for "Wireless microphones in the $25 \mathrm{MHz}$ to $3 \mathrm{GHz}$ frequency range." The ETSI Specialist Task Force (STF) 386 was founded to introduce cognitive procedures into the existing standards. The STF 386 is currently in the $3^{\text {rd }}$ phase, where RF tests with measurements from a technology demonstrator should be carried out, which will be supported in the frame of this project.

Relevant Technical Committees (TC) for standardization at ETSI are the TC Reconfigurable Radio Systems (TC RRS) with the sub-groups on System Aspects (RRS 01), Reconfigurable Radio Equipment Architecture (RRS 02), Cognitive Management and Control (RRS 03) and Public Safety (RRS 04), the TC EMC, and Radio Spectrum Matters (TC ERM) with the sub-groups on Wideband Data Systems (TG 11), Radio Microphones, Cordless Audio and Audio Links (TG 17 WG 3) and Generic Short Range Devices (TG 28) as well as the TC Broadband Radio Access Networks (TC BRAN). The work on STF 386 is running in TC ERM TG17 WG3. Until now, some Technical Reports (TR) and Specifications (TS) have been finished or are in preparation by the listed TCs.

On world-wide level, the Standards Coordinating Committee 41 (SCC41) within IEEE is developing standards related to dynamic spectrum access networks. The focus is on improved use of spectrum. New techniques and methods of dynamic spectrum access require managing interference, coordination of wireless technologies and include network management and information sharing. 
Currently six working groups, IEEE 1900.1 - 1900.6, plus one ad hoc group have been established by SCC41, the IEEE 1900.1 Working Group on Definitions and Concepts for Dynamic Spectrum Access: Terminology Relating to Emerging Wireless Networks, System Functionality, and Spectrum Management, the

IEEE 1900.2 Working Group on Recommended Practice for the Analysis of In-Band and Adjacent Band Interference and Coexistence between Radio Systems, the

IEEE 1900.3 Working Group on Recommended Practice for Conformance Evaluation of Software Defined Radio (SDR) Software Modules, the

IEEE 1900.4 Working Group on Architectural Building Blocks Enabling Network-Device Distributed Decision Making for Optimized Radio Resource Usage in Heterogeneous Wireless Access Networks, the

IEEE 1900.5 Working Group on Policy Language and Policy Architectures for Managing Cognitive Radio for Dynamic Spectrum Access Applications, the

IEEE 1900.6 Working Group on Spectrum Sensing Interfaces and Data Structures for Dynamic Spectrum Access and other Advanced Radio Communication Systems and the IEEE SCC41 ad hoc on White Space Radio.

Additionally, the IEEE 802.19 Wireless Coexistence Working Group develops standards for coexistence between wireless standards of unlicensed devices and the IEEE 802.22 Working Group on Wireless Regional Area Networks is working on standards for enabling the rural broadband wireless access using CR technology in TV Whitespaces.

\section{THE C-PMSE PROJECT}

\section{A. Overview}

The consortium of the C-PMSE project consists out of six partners from industry, one research institute, and three universities. The PMSE industry is represented by beyerdynamic $\mathrm{GmbH} \& \mathrm{Co}$. KG, Robert Bosch $\mathrm{GmbH}$, and Sennheiser electronic GmbH \& Co. KG. Huawei Technologies Düsseldorf $\mathrm{GmbH}$ as mobile communication device manufacture and network technology provider contributes its strong expertise in mobile and nomadic communications. The Institut für Rundfunktechnik $\mathrm{GmbH}$, a research institute founded by German, Austrian, and Swiss public broadcasters, represents the broadcasters as users of PMSE technology and the broadcasting services (like DVB-T) as primary user of frequency spectrum in the TV bands. The eesy-id $\mathrm{GmbH}$ is involved to develop the sensor nodes, and the RFmondial $\mathrm{GmbH}$ will develop the monitoring software and data base infrastructure. The universities Friedrich-Alexander-University Erlangen-Nürnberg, Gottfried Wilhelm Leibniz University Hannover, and Ruhr University Bochum provide the practical and scientific expertise in electronics, signal processing, software defined radio, cognitive radio, reactive and proactive radio resource management, signal classification, measurements and modeling to the consortium.

The C-PMSE project is started on April, 1st. 2011 and will run in a first phase for 26 month.

\section{B. Aims and Objectives}

The C-PMSE project has two major objectives. The first is setting up a research platform in the labs of the universities in Hannover and Erlangen for lab trials, and a lager one at the fair grounds and exhibition halls of "Messe Berlin" for field trials. The second is to drive standardization and regulation for cognitive PMSE systems.

Beside of the realization and setup of the lab and field trial platforms, the major goals include the development of all necessary hardware and software components, the development and installation of a distributed scanning system with signal processing, network and data base infrastructure, and the verification of feasibility, and finally the functional demonstration in compliance with activities of the working group ETSI STF 386 on the lab and field trial platforms. Additionally, coexistence investigations with other wireless services and devices, like white space devices, mobile communication, terrestrial digital video broadcasting (DVB-T), will be performed. The field trial platform will remain at Messe Berlin after the end of the project and is intended to be used for further field tests and follow up projects.

Standardization and regulation have the same priority in this project. The major goal is to consider cognitive radio (CR)enabled approaches for PMSE applications in worldwide regulatory frameworks and standardization activities. EU member state administrations are currently discussing how cognitive technologies can be used for spectral efficiency in the field of broadband applications generally. This is also one of the major topics to be discussed for the Radio Spectrum Policy Program (RSPP) in Europe. On the other hand, the PMSE industry states that some basic ideas and methodologies of CR are not suitable for the requirements of PMSE, like stand-alone sensing solutions, use of cognitive pilot channels, and alwaysavailable access to data bases. A key objective of the C-PMSE project is therefore, to provide appropriate and validated inputs for standardizations and frequency regulations.

Planned activities within the frame of the C-PMSE project will provide inputs for regulation at ECC WG FM45 and WG SE43 as well as inputs for standardization at ETSI TC RSS, TC ERM and TC BRAN. Further on, inputs will be provided for WRC-12 concerning agenda item 1.5 and 1.19 at ECC CPG-A and CPG-B and at the WRC-12 conference itself. If necessary and possible advanced inputs are given for WRC-15 agenda items.

\section{Research Plattform for Evaluation \& Validation}

The research platform follows the fundamental concept provided by the ETSI STF 386 in the technical specification TS 102800 .

The key elements of the concept of lab and field trial platforms are a Scanning Receiver Subsystem (SCS), a local Frequency Coordinator (FCO), and a cognitive-enabled PMSE base station (BS) and adaptable PMSE mobile stations (MS).

The SCS consists of one or multiple Scanning Receiver Controller (SCC) and one or multiple Scanning Receiver (SCR). An SCC is in charge of managing SCRs connected to it. The SCC is scheduling the scanning jobs to be carried out by 
the SCRs, performing preprocessing (signal detection, signal classification, parameter extraction) of data acquired and delivering the extracted information to the Cognitive Engine (CEN) of BS, which requested the SCC to perform the actions. To establish preprocessing in the SCCs allows to distribute the computational load of the required signal processing and to significantly reduce the data-rate requirement of the interface between SCS and CEN.

A FCO is a local entity for spectrum portfolio management, so it tells the cognitive-enabled PMSE systems, which frequency bands are available for PMSE services. Therefore, the FCO is connected to regulatory data bases and via an interface to the CEN. Depending on the availability of such databases, we might establish our own geo-location based regulatory database with the most important features required.

The PMSE base station contains a Cognitive Engine (CEN), a Performance Monitor (PMO), a Service Level Monitor (SLM), a Radio Resource Manager (RRM), and adaptable PMSE radio links to the Mobile Stations (MS), e.g. wireless microphones or in-ear monitors.

The CEN represents the artificial intelligence of the system and is realized by four engines running in parallel. The first is the Fusion Engine (FEN), which merges all information acquired via the SCS or provided by the FCO to generate a Radio Environment Map (REM), which is transferred to the RRM. The second is the Optimization Engine (OEN), which searches based on the REM, metrics of risk and quality assessments, and PMSE link parameter sets for different options of resource allocations within the given spectrum portfolio. The third process, the Decision-making Engine (DEN) selects out of all possible optimizations the one most suitable for the next reallocation of resources. Beside this, the DEN keeps always updated fallback configurations (panic action set) of the resource allocations available, which can be employed if an outage by unforeseeable distortions is noticed or awaited. The fourth process is the Cyclic Unit (CYU), which is the central controller and scheduler of the CEN and the whole BS, so that all processes are timely in line.

The PMO allows tracing the behavior and status of the BS. It generates a log file, which can be visualized in the so-called Demonstration Monitor (DMO), which is the control center of all entities (FCO, all SCS, all PMSE BSs) of the lab and field trial platforms. The DMO also provides advanced storage capabilities to store measurement data and log files. The service level required for PMSE links can be adjusted via the Service Level Entry (SLE) and is traced by the Service Level Monitor (SLM). The requested service level influences the solutions discovered by the CEN.

The RRM hold the REM and the Link Parameter Sets (LPS), which contain information on frequency allocations, power allocations, adaptive modulation and coding schemes chosen and devices installed. The Action Sequencer (ASQ) acts based on instructions by the CEN. It is in charge of delivering commands and corresponding LPSs to the PMSE radio links.

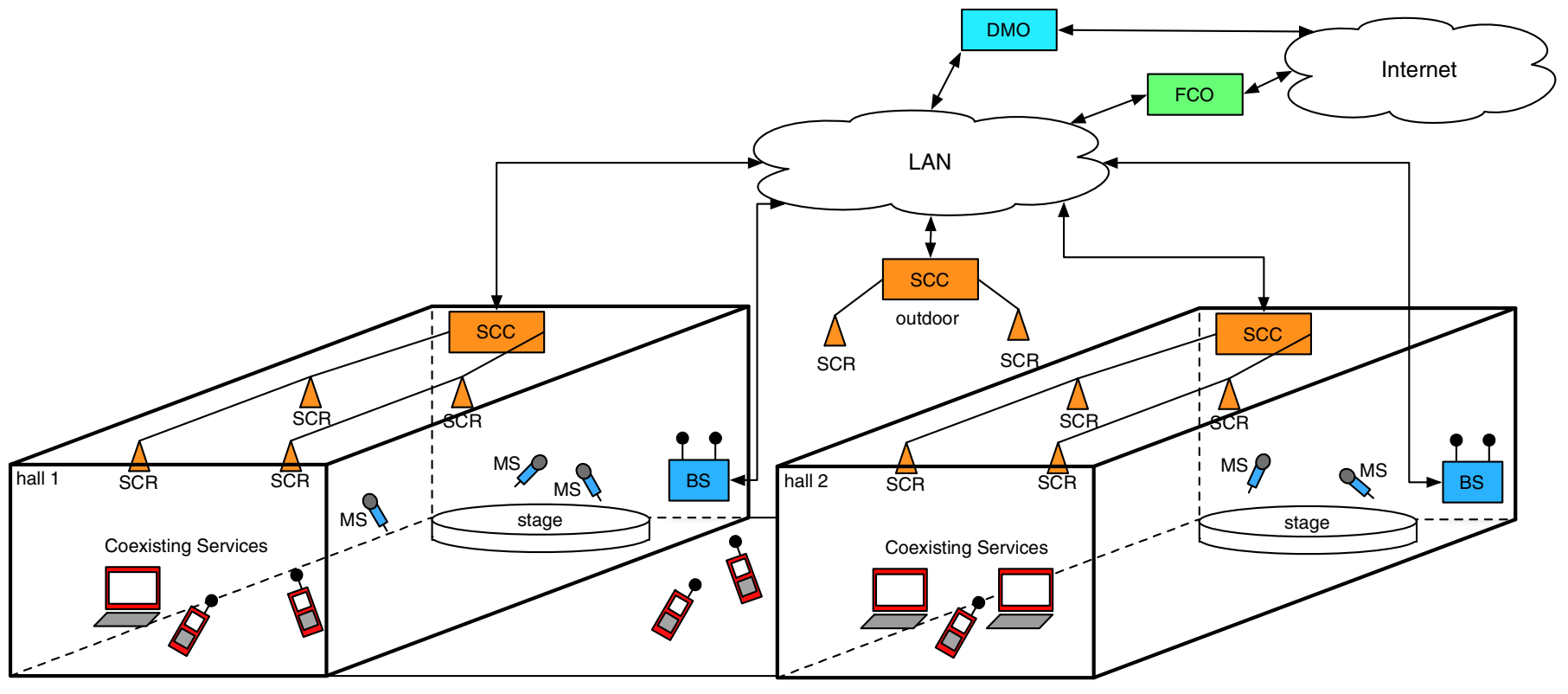

Fig. 1: General overview of the field trial platform

SCR: Scanning Receiver

SCC: Scanning Controller

DMO: Demonstration Monitor

FCO: Frequeny Coordinator
MS: PMSE Mobile Station

BS: PMSE Base Station

LAN: Local Area Network 
To connect with different kinds of demonstrators and prototypes the lab and field trial platforms will have a common interface to demonstration hardware and prototypes. The specification of this interface is not part of the work conducted in the ETSI STF 386, but required for the field and lab trials.

Depending on availability, coexisting devices and services (e.g. Legacy PMSE, DVB-T2, ECMA 392, IEEE 802.11af, LTE Advanced) will be placed as products, prototypes, or as demonstrators based on signal generator or software defined radio realizations into the measurement trials.

It is planed that the two lab trial platforms, which are also used for early system tests during developments, will have only a single SCC connected with four SCRs. The field trial platform will be a fixed installation of the SCS with ten SCCs and 50 SCCs distributed among the buildings and the fair grounds in Berlin. It is aimed to gather measurement data of several events. The measurements and results will be made available to interested parties of academics, industry, and standardization, and regulation bodies.

Fig. 1 provides a general overview to the field trial platform and its components. Multiple SCR and a SCC will be mounted under the ceiling of an exhibition hall. The BS of each exhibition hall is maintaining the PMSE links to one or multiple MSs. Coexisting services like nomadic wireless communications, cellular communications, and short range communications might occur within a hall, in other halls or outside. The multi-sensor, multi-location SCS allows to detect possible interferes before they take seriously effect, for example by extracting the directions of movements of interferes and proactive adjustments of the frequency allocations or by switching to more robust link parameter sets.

The interconnection of the C-PMSE entities is outlined in Fig. 1. Each set of SCRs and a single SCC will be part of their own interconnection plane, while SCC, BS, DMO, and FCO are connected to the Local Area Network (LAN), which is possibly realized as Virtual LAN (VLAN). An Internet connection is required for the $\mathrm{FCO}$ to request and receive information regulation data bases and available for the DMO for remote monitoring and control.

\section{OUTLOOK AND CONCLUSION}

The fixed installation of a SCS is unique and will enable an evaluation and validation of multi-sensor, multi-location cognitive radio approaches, not limited, but focused to PMSE applications and other coexisting services in the Ultra High Frequency (UHF) band and especially in the TV bands. With the C-PMSE project, application-oriented research including modeling, evaluation, and validation of heterogeneous coexistence scenarios are established side-by-side with strong activities in frequency regulation and standardization in Europe and worldwide. Especially adequate models, evaluated approaches, identified limitations and verified research results will provide resilient facts for the product development and for the frequency regulation and standardization of cognitive technologies.

\section{ACKNOWLEDGMENT}

The C-PMSE project is co-funded by the German Federal Ministry of Economics and Technology (BMWi).

\section{REFERENCES}

[1] ETSI ERM / TG17 WP3, ETSI Specialist Task Force STF 386: „methods, parameters and test procedures for cognitive interference mitigation techniques for PMSE (Programme Making and Special Events) devices"

[2] Prasad, R.; Pawelczak, P.; Hoffmeyer, J. \& Berger, H. "Cognitive Functionality in Next Generation Wireless Networks: Standardization Efforts", Communications Magazine, IEEE, 2008, 46, 72-78

[3] Mueck, M.; Piipponen, A.; Kallioja rvi, K.; Dimitrakopoulos, G.; Tsagkaris, K.; Demestichas, P.; Casadevall, F.; Pérez-Romero, J.; Sallent, O.; Baldini, G.; Filin, S.; Harada, H.; Debbah, M.; Haustein, T.; Gebert, J.; Deschamps, B.; Bender, P.; Street, M.; Kandeepan, S.; Lota, J.; Hayar, A.; , "ETSI reconfigurable radio systems: status and future directions on software defined radio and cognitive radio standards," Communications Magazine, IEEE, vol.48, no.9, pp.78-86, Sept. 2010

[4] Cordeiro, C.; Challapali, K.; Birru, D.; Sai Shankar, N.; , "IEEE 802.22: the first worldwide wireless standard based on cognitive radios," New Frontiers in Dynamic Spectrum Access Networks, 2005. DySPAN 2005. 2005 First IEEE International Symposium on, pp.328-337, 8-11 Nov. 2005

[5] Raychaudhuri, D.; Jing, X. "A Spectrum Etiquette Protocol for Efficient Coordination of Radio Devices in Unlicensed Bands",14th IEEE Conference on Personal, Indoor and Mobile Radio Communications, PIMRC, China, 2003

[6] Srikanteswara, S.; Choudhury, D.; , "A review of TV Whitespace portable devices," Radio and Wireless Symposium (RWS), 2010 IEEE, pp.480-483, 10-14 Jan. 2010

[7] EU workshop on "A long term approach to radio spectrum for PMSE in Europe", 26 October 2009, Summary report, (http://ec.europa.eu/information society/policy/ecomm/radio spectrum/t opics/reorg/dividend/pmse/index en.htm) 